\title{
Healing the mucosa in Crohn's disease: does it matter?
}

\author{
Khoon Lin Ling ${ }^{1}$, MBBS, MRCP, Sai Wei $\underline{\text { Chuah }}^{1}$, MBChB, MRCP, San Choon Kong $^{1}$, MBBS, MRCP
}

ABSTRACT Clinical remission has been the therapeutic goal of Crohn's disease treatment for many years. While it has helped to ameliorate the symptoms, this treatment strategy has not brought about significant changes in the need for abdominal surgery in the natural history of Crohn's disease. The advent of biological agents (biologics) has shown that it is possible to induce and maintain mucosal healing in a significant proportion of treated patients. Data is also emerging to show that this has translated to fewer instances of hospitalisation and surgery for these patients. This is a paradigm shift in the therapeutic goal of Crohn's disease treatment.

Keywords: Crohn's disease, drug therapy, mucosal healing

\section{INTRODUCTION}

Crohn's disease (CD) is a chronic inflammatory disease of the gastrointestinal $(\mathrm{Gl})$ tract. Patients with $\mathrm{CD}$ present with a host of symptoms, including diarrhoea, haematochezia, abdominal pain, weight loss and fever. The last few decades have seen a gradual increase in the number of drugs available for use in the treatment of $\mathrm{CD}$. From a time when only sulfasalazine, steroids and antibiotics were used, we now have immunomodulators such as thiopurines and methotrexate, as well as biological agents. While these newer drugs are effective for inducing clinical remission in $\mathrm{CD}$, data have recently emerged to show that they are also effective in reducing complications, and consequently, the need for surgery in $C D$ patients.

\section{INTESTINAL ULCERS AND INFLAMMATION IN CROHN'S DISEASE}

Chronic intestinal inflammation causes Gl ulcers in CD. This is a transmural inflammation. ${ }^{(1)}$ The intact Gl mucosa is a vital part of the mucosal barrier that separates the luminal microbiota from the mucosal immune system. In the intestines, components of the mucosal barrier include the intact epithelial cell layer, tight junctions, and antimicrobial peptides such as defensins, which are produced by the intestinal mucosa. Defects in different components of this barrier have been found in patients with inflammatory bowel disease (IBD). For example, it has been suggested that altered defensin production plays an important role in the pathogenesis of IBD. ${ }^{(2)}$ Similarly, defects in proteins that make up the tight junctions, such as the occludins and claudins, have also been described in patients with IBD. These defects are believed to cause breaks in the mucosal barrier, which permit the passage of microorganisms into the mucosa and submucosa. There, the microorganisms meet and activate the mucosal immune system, causing chronic intestinal inflammation.

An altered mucosal immune response has also been shown in animal models and IBD patients. ${ }^{(3-5)} \mathrm{A}$ relative deficiency of immune suppressor cells (e.g. regulatory $T$ cells) have been found in IBD patients. ${ }^{(6)}$ Differences in the way antigen-presenting cells and effector $\mathrm{T}$ cells respond to bacterial stimuli have also been shown in IBD patients, compared to normal controls. ${ }^{(7)}$ One way by which this is mediated is through differences in the way the pattern recognition receptor NOD2/CARD15 recognises microorganisms in IBD patients versus healthy controls. ${ }^{(8)}$ The NOD2/CARD15 gene is the first IBD gene to be discovered.

\section{THERAPEUTIC ENDPOINTS FOR CROHN'S DISEASE}

The target of gastroenterologists in treating patients has not changed for many years..$^{(9)}$ Although endoscopic, histological and radiological proof is required before a diagnosis of IBD can be made, gastroenterologists often treat $C D$ patients only to relieve symptoms and achieve biochemical remission. The aim is not to achieve complete healing of the ulcers. This, however, is not unique to routine clinical practice. For instance, clinical trials examining the efficacy of novel therapies make use of disease activity indices, such as the Crohn's disease activity index (CDAl), which measure clinical symptoms and not endoscopic disease activity.

Until the advent of infliximab in the 1990s, it was not uncommon to find significant ulcers in CD patients in clinical and biochemical remission after a course of steroids. Trials of biological agents since the 1990s have taught us that a proportion of patients in clinical and biochemical remission, as defined by CDAI, also achieve complete healing of mucosal ulcers. ${ }^{(10)}$ The impressive images of ulcer healing in patients who had received only one dose of infliximab demonstrated that contrary to what was often seen in CD patients with steroid-induced remission, it was possible for a significant proportion of patients to achieve ulcer healing.

\section{MUCOSAL HEALING AND ENDOSCOPIC SCORING INDICES}

Complete mucosal healing can be simplistically defined as the complete absence of ulcers and inflammation. However, since

${ }^{1}$ Department of Gastroenterology and Hepatology, Singapore General Hospital, Singapore

Correspondence: Dr Ling Khoon Lin, Senior Consultant, Department of Gastroenterology and Hepatology, Singapore General Hospital, Outram Road, Singapore 169608. ling.khoon.lin@sgh.com.sg 
this is difficult to achieve even in patients on biological agents, it would be useful to define partial mucosal healing. Endoscopic activity indices would enable one to grade different degrees of mucosal healing - from complete healing to absence of mucosal healing. A number of endoscopic scoring indices for $C D$ have been devised and used in different drug trials. The Crohn's disease endoscopic index of severity (CDEIS) calculates the percentage of involvement of different ileocolonic segments. ${ }^{(11)}$ It is complex to use, requires training and experience for estimating the extent of ulcerated or diseased mucosal surfaces, as well as experience in distinguishing deep from superficial ulceration. While some studies have demonstrated good correlation between CDEIS (which measures mucosal activity) and CDAI (which measures clinical symptoms), others have shown poor correlation between the two indices. ${ }^{(11-14)}$ As the complexity of CDEIS limits its usefulness in clinical practice, its use is largely restricted to a clinical trial setting. The Simple Endoscopic Score for Crohn's Disease (SES-CD) includes four variables - ulcer size, extent of ulcerated surface, extent of affected surface, and stenosis - in five segments of the bowel. ${ }^{(15)}$ SES-CD correlates well with CDEIS. Although it is now possible to describe mucosal lesions in a consistent manner using either CDEIS or SES-CD, there is still no commonly agreed cutoff value in either scoring system for defining endoscopic response to treatment, endoscopic remission or mucosal healing.

Of all the CD endoscopic indices, the Rutgeerts score is the only postoperative endoscopic grading system that has been used to describe the severity of endoscopic recurrence at the ileocolic anastomosis and preanastomotic ileum post resection. ${ }^{(16)}$ Scoring is based on the presence of ulcers, ileitis, nodularity and strictures. Grading ranges from i0 to i4. Although the reproducibility of the Rutgeerts scale has not been prospectively validated, the severity of endoscopic lesions at one-year post operation has been demonstrated to be predictive of clinical recurrence. Postoperative recurrence was initially defined as Rutgeerts score i1, but most clinical trials have used i2 as the cutoff to define endoscopic recurrence. In a recent study, the preexisting Rutgeerts endoscopic score was modified to incorporate a scoring system of colonic lesions after surgery. ${ }^{(17)}$

\section{CLINICAL SIGNIFICANCE OF MUCOSAL HEALING}

When contemplating the use of mucosal healing as a therapeutic goal, it is important for both the clinician and patient to consider whether the presence of ulcers can predict future disease activity and future need for hospitalisation or major abdominal surgery, as well as whether mucosal healing will modify the natural history of IBD. This is especially important in patients who have already undergone their first abdominal surgery. ${ }^{(18)}$

Allez et al have shown that the presence of deep ulcerations in $\mathrm{CD}$ patients was a risk factor for penetrating complications and surgery. ${ }^{(19)}$ Among patients with severe endoscopic lesions in their cohort, the probability of colectomy was $31 \%, 42 \%$, and $62 \%$ at one, three and eight years, respectively, whereas in the absence of severe endoscopic lesions, the probability of colectomy was $6 \%, 8 \%$ and $18 \%$ at the same time points. A study of postoperative patients revealed that CDAI could discriminate between those with and without endoscopic recurrence in only $65 \%$ of cases, suggesting that CDAl alone was inadequate for the detection of postoperative recurrence of $\mathrm{CD} .{ }^{(20)}$ These findings were supported by a small, randomised controlled study by Regueiro et al, which found poor correlation between postoperative endoscopic recurrence and clinical symptoms, CDAI and C-reactive protein. ${ }^{(21)}$

\section{ALTERING THE NATURAL HISTORY OF IBD}

$C D$ is characterised by a relapsing and remitting disease course. Medical therapy is required by the majority of patients in order to maintain remission, coupled with intermittent induction therapy to manage flares. Just over $80 \%$ of Singaporean patients present with inflammatory, non-stricturing, non-penetrating disease. ${ }^{(22)}$ In the long term, inflammation results in stricturing and penetrating disease in $50 \%$ of all CD patients. About $50 \%$ of patients with either penetrating or stricturing disease require major abdominal surgery within six months of developing the disease. Even after the first abdominal surgery, $C D$ often recurs either at or proximal to the anastomosis. Up to $70 \%$ of patients require reoperation within ten years. ${ }^{(23)}$ Risk factors for surgery include smoking, young age at diagnosis, perianal disease and the need for steroids at initial diagnosis. Such a disease course is obviously disabling and has significant morbidity. It would, therefore, be important for both the patient and society to delay or interrupt the natural history of CD.

Although evidence for this is still sparse, directly targeting tissue damage with treatments that induce and maintain mucosal healing in the early stage of disease may reduce complications and alter or halt the progression of disease. As endoscopic assessment provides objective evidence of mucosal healing or damage, endoscopic outcomes are being increasingly used as efficacy endpoints in clinical trials. In the short term, mucosal healing has been associated with reductions in CDAI and reduced steroid use. ${ }^{(24)}$ In the longer term, mucosal healing has been shown to be associated with durable remission, lower complication rates and reduced need for surgery and hospitalisation. ${ }^{(25,26)}$ Although mucosal healing is associated with improved outcomes, there is currently insufficient evidence to corroborate that treatment escalation to induce mucosal healing results in improved outcomes.

\section{DRUGS AND MUCOSAL HEALING}

Not all drugs are equally effective in inducing and maintaining mucosal healing in CD. We present below a description of the common drugs used and their efficacies in the treatment of CD.

\section{Steroids}

Corticosteroids are effective suppressors of inflammation and can induce clinical remission. $60 \%$ of patients have a complete clinical response to steroids, while $30 \%$ have partial response and 
$10 \%$, no response. ${ }^{(27,28)}$ Only $10 \%$ of patients will have complete or partial mucosal healing at the end of their course of steroids, and only $30 \%$ of patients will have a prolonged clinical response to the initial course of steroids at one year. ${ }^{(28)}$ It is clear from multiple clinical trials that corticosteroids - both prednisolone and budesonide - are of no benefit in maintaining clinical remission or preventing new flares, and they do not alter the natural history of the disease. They are also not useful for the maintenance of mucosal healing.

\section{Thiopurines}

Thiopurines (azathioprine and 6-mercaptopurine) have been shown to induce clinical and endoscopic remission. Among CD patients who achieved clinical remission in D'Haens et al's study, $70 \%$ of those with colonic disease and $54 \%$ with ileal disease had complete mucosal healing. ${ }^{(29)}$ Another study, which consisted of 53 Japanese patients, reported a complete clinical response in $23 \%$ of the patients, with $42 \%$ showing clinical improvement. ${ }^{(30)}$ Colonoscopy done before and after azathioprine treatment for the Japanese cohort showed complete mucosal healing in $56 \%$, partial mucosal healing in $19 \%$ and no healing in $25 \%$ of the patients.

\section{Methotrexate \\ Methotrexate is often used as the second immunomodulator when patients stop thiopurines because of treatment failure or adverse effects. In CD, methotrexate has been shown to induce and maintain clinical remission in placebo-controlled randomised trials. An early study has shown that $36 \%$ of CD patients achieve mucosal healing. ${ }^{(31)}$ Laharie et al, who compared mucosal healing induced by methotrexate, azathioprine and infliximab in a single- centre study, found that mucosal healing was achieved in $11 \%$, $50 \%$ and $60 \%$ of patients, respectively. ${ }^{(32)}$}

\section{Biologics}

Biologics, also known as biological agents, have been the most potent agents in inducing clinical and endoscopic remission in CD patients. Van Dullemen et al reported dramatic endoscopic improvement in patients given just a single dose of infliximab. ${ }^{(10)}$ Subsequent trials with infliximab, adalimumab and certolizumab have demonstrated the efficacy of anti-tumour necrosis factor (TNF) agents in inducing mucosal healing in CD patients. The ACCENT 1 (A Crohn's Disease Clinical Trial Evaluating Infliximab in a New Long-term Treatment Regimen) endoscopic substudy showed that over $40 \%$ of patients who received infliximab regularly had complete mucosal healing compared with $18 \%$ of patients who did not. ${ }^{(33,34)}$ In the SONIC (Study of Biologic and Immunomodulator Naive Patients in Crohn's Disease) trial, $30 \%$ of patients on infliximab monotherapy achieved mucosal healing compared with $44 \%$ of patients on combined azathioprine and infliximab therapy. ${ }^{(35)}$ The EXTEND (Extend the Safety and Efficacy of Adalimumab Through Endoscopic Healing) placebocontrolled trial evaluated the impact of adalimumab on mucosal healing in patients with active ileocolonic CD. ${ }^{(36)}$ Patients received open-label adalimumab $160 \mathrm{mg}$, then $80 \mathrm{mg}$ induction therapy at Weeks 0 and 2, and were then randomised at Week 4 to maintenance therapy with fortnightly adalimumab $40 \mathrm{mg}$ or placebo. Mucosal healing at Week 12 was seen in $27 \%$ of the treatment group compared to $13 \%$ in the placebo group. At Week 52, mucosal healing was demonstrated in $24 \%$ and $0 \%$ of patients in the treatment and placebo arms, respectively. ${ }^{(36)}$ The MUSIC (endoscopic MUcoSal Improvement in patients with active $C D$ treated with certolizumab pegol) trial studied mucosal healing in patients treated with certolizumab pegol. ${ }^{(37)}$ Patients on subcutaneous certolizumab pegol showed improvement in the endoscopic appearance of mucosal ulcers at both Weeks 10 and 54. However, complete mucosal healing was only seen in a small percentage of patients $-4 \%$ and $8 \%$ at Week 10 and Week 54, respectively. ${ }^{(37)}$

\section{NONINVASIVE METHODS OF ASSESSING MUCOSAL DISEASE ACTIVITY}

In the vast majority of clinical trials, mucosal healing was assessed using colonoscopy with ileoscopy. However, endoscopy is expensive and associated with the risk of perforation. It is also difficult to justify to an asymptomatic patient the need for regular ileocolonoscopy. If the attainment of complete mucosal healing is to play a greater role in the evaluation of therapeutic response to drugs, there will be a need for noninvasive surrogate markers to quantify asymptomatic mucosal disease activity, so as to allow serial monitoring. Regular monitoring with endoscopy or noninvasive surrogate markers can then identify patients with active mucosal disease so that appropriate treatment escalation can be instituted.

\section{Stool calprotectin}

Calprotectin, a marker of inflammation, is produced by intestinal epithelial cells and leukocytes. It is a heterodimer made up of S100A8 and S100A9. Elevated levels of stool calprotectin have been found in IBD patients, and this has been shown to correlate with endoscopic disease activity measured using CDEIS and SES-CD. ${ }^{(38,39)}$ Stool calprotectin levels have also been found to decrease with anti-TNF therapy-induced endoscopic mucosal improvement. The reduction in stool calprotectin with anti-TNF therapy is found to correlate with endoscopic mucosal healing as measured by CDEIS. ${ }^{(40)}$ The levels seldom normalise to that seen in normal controls, suggesting ongoing subclinical inflammatory activity even in the absence of mucosal lesions. ${ }^{(41)}$ The value of stool calprotectin in predicting future clinical activity has been studied. In the STORI (infliximab diSconTinuation in CrOhn's disease patients in stable Remission on combined therapy with Immunosuppressors) study, stool calprotectin levels were higher in patients who relapsed than in patients who had sustained remission. ${ }^{(42)}$ A normal stool calprotectin level had a high negative predictive value for a flare of disease activity, for up to three months. Predictive accuracy diminishes beyond three months. 
In the post-surgical setting, it has been suggested that a stool calprotectin value of more than $200 \mathrm{mg} / \mathrm{L}$ is predictive of early mucosal disease recurrence. ${ }^{(43)}$

\section{Noninvasive cross-sectional imaging}

Computed tomographic enterography and magnetic resonance enterography (MRE) have been used in the initial assessment of disease extent and activity in patients with $C D$, and as tools to follow up on disease activity after the initiation of therapy. ${ }^{(44)}$ While both modalities have been found to be useful, MR imaging has an advantage in that it does not expose the patient to ionising radiation, and can therefore be used repeatedly to follow up on the resolution of disease activity. Rimola et al have described an MR activity index for CD activity that correlates well with CDEIS, and this index has been validated. ${ }^{(45-47)}$ In the postoperative setting, MRE has $100 \%$ sensitivity and $89 \%$ specificity in detecting postoperative recurrence, and current evidence suggests that it may be as good as endoscopy. ${ }^{(48)}$

Stool calprotectin and MRE are useful noninvasive tests that can be performed on patients who cannot or will not undergo regular endoscopy to monitor for mucosal healing. It is conceivable that algorithms will be established where a CD patient is monitored for mucosal healing using a mixture of endoscopy, stool calprotectin and MRE in the not-too-distant future.

\section{CONCLUSION}

The goal of treatment strategies for $\mathrm{CD}$ has traditionally been clinical remission and resolution of symptoms. The advent of more efficacious treatment modalities means that in addition to symptom resolution, it is now possible to aim for healing of intestinal ulcers. Randomised trials have demonstrated that immunomodulators and biological agents can induce and maintain clinical remission and mucosal healing. Mucosal healing is associated with several benefits, including steroid-free remission, improved quality of life, reduced hospitalisation and decreased need for surgery. Mucosal healing is still largely assessed using endoscopy, an invasive procedure. However, there is current evidence that certain noninvasive tests correlate well with mucosal disease activity. The results of tests such as stool calprotectin and MRE have been shown to correlate with endoscopic activity, although these noninvasive tests are not widely available.

Several questions about mucosal healing remain unanswered. While it would be ideal to examine patients with endoscopy at regular intervals to monitor for mucosal healing, the procedure is expensive and associated with a risk of adverse events. It is still unknown when and how often endoscopy should take place after the initiation of a new therapy or post-surgery. Whether the escalation of therapy in asymptomatic patients with evidence of ongoing mucosal inflammation would be of any benefit is yet to be ascertained. It is also unknown if treatment de-escalation would be possible in patients who have demonstrated both clinical remission and complete mucosal healing. The coming years should see the completion of clinical trials that may unravel some of these questions, which will help physicians decide on the most appropriate treatment strategies for patients with CD.

\section{REFERENCES}

1. Xavier RJ, Podolsky DK. Unravelling the pathogenesis of inflammatory bowel disease. Nature 2007;448: 427-34.

2. Wehkamp J, Schmid M, Fellermann K, Stange EF. Defensin deficiency, intestinal microbes, and the clinical phenotypes of Crohn's disease. J Leukoc Biol 2005; 77:460-5.

3. Neurath MF, Finotto S. Translating inflammatory bowel disease research into clinical medicine. Immunity 2009; 31:357-61.

4. Neurath MF, Finotto S, Glimcher LH. The role of Th1/Th2 polarization in mucosal immunity. Nat Med 2002; 8:567-73.

5. Abraham C, Cho JH. IL-23 and autoimmunity: new insights into the pathogenesis of inflammatory bowel disease. Annu Rev Med 2009; 60:97-110.

6. Boden EK, Snapper SB. Regulatory T cells in inflammatory bowel disease. Curr Opin Gastroenterol 2008; 24:733-41.

7. Abraham C, Medzhitov R. Interactions between the host innate immune system and microbes in inflammatory bowel disease. Gastroenterology 2011; 140:1729-37.

8. Cooney R, Baker J, Brain O, et al. NOD2 stimulation induces autophagy in dendritic cells influencing bacterial handling and antigen presentation. Nat Med 2010; 16:90-7.

9. Rutgeerts P, Vermeire S, Van Assche G. Mucosal healing in inflammatory bowel disease: impossible ideal or therapeutic target? Gut 2007; 56:453-5.

10. van Dullemen HM, van Deventer SJ, Hommes DW, et al. Treatment of Crohn's disease with anti-tumor necrosis factor chimeric monoclonal antibody (cA2). Gastroenterology 1995; 109:129-35.

11. Mary JY, Modigliani R. Development and validation of an endoscopic index of the severity for Crohn's disease: a prospective multicentre study. Groupe d'Etudes Therapeutiques des Affections Inflammatoires du Tube Digestif (GETAID). Gut 1989; 30:983-9.

12. Landi B, Anh TN, Cortot A, et al. Endoscopic monitoring of Crohn's disease treatment: a prospective, randomized clinical trial. The Groupe d'Etudes Therapeutiques des Affections Inflammatoires Digestives. Gastroenterology 1992; 102:1647-53.

13. Modigliani R, Mary JY, Simon JF, et al. Clinical, biological, and endoscopic picture of attacks of Crohn's disease. Evolution on prednisolone. Groupe d'Etude Therapeutique des Affections Inflammatoires Digestives. Gastroenterology 1990; 98:811-8.

14. Rutgeerts P, Diamond RH, Bala M, et al. Scheduled maintenance treatment with infliximab is superior to episodic treatment for the healing of mucosal ulceration associated with Crohn's disease. Gastrointest Endosc 2006; 63:433-42.

15. Daperno M, D'Haens G, Van Assche G, et al. Development and validation of a new, simplified endoscopic activity score for Crohn's disease: the SESCD. Gastrointest Endosc 2004; 60:505-12.

16. Rutgeerts P, Geboes K, Vantrappen G, etal. Predictability of the postoperative course of Crohn's disease. Gastroenterology 1990; 99:956-63.

17. Marteau P, Lémann M, Seksik $P$, et al. Ineffectiveness of Lactobacillus johnsonii LA1 for prophylaxis of postoperative recurrence in Crohn's disease: a randomised, double blind, placebo controlled GETAID trial. Gut 2006; 55:842-7.

18. Rutgeerts P, Geboes K, Vantrappen G, et al. Natural history of recurrent Crohn's disease at the ileocolonic anastomosis after curative surgery. Gut 1984; 25:665-72.

19. Allez M, Lemann M, Bonnet J, et al. Long term outcome of patients with active Crohn's disease exhibiting extensive and deep ulcerations at colonoscopy. Am J Gastroenterol 2002; 97:947-53.

20. Viscido A, Corrao G, Taddei G, Caprilli R. "Crohn's disease activity index" is inaccurate to detect the post-operative recurrence in Crohn's disease. A GISC study. Gruppo Italiano per lo Studio del Colon e del Retto. Ital J Gastroenterol Hepatol 1999; 31:274-9. 
21. Regueiro M, Kip KE, SchrautW, et al. Crohn's disease activity index does not correlate with endoscopic recurrence one year after ileocolonic resection. Inflamm Bowel Dis 2011; 17:118-26.

22. Tay WL, Thia KTJ, Allen JC, et al. The risk of Crohn's disease progression towards intestinal complications in an Asian cohort. Gastroenterology 2011; 140 (Suppl 1):S783.

23. Oriuchi T, Hiwatashi N, Kinouchi Y, et al. Clinical course and longterm prognosis of Japanese patients with Crohn's disease: predictive factors, rates of operation, and mortality. J Gastroenterol 2003; 38:942-53.

24. Baert F, Moortgat L, Van Assche G, et al. Mucosal healing predicts sustained clinical remission in patients with early-stage Crohn's disease. Gastroenterology 2010; 138:463-8.

25. Schnitzler F, Fidder $H$, Ferrante $M$, et al. Mucosal healing predicts longterm outcome of maintenance therapy with infliximab in Crohn's disease. Inflamm Bowel Dis 2009; 15:1295-301.

26. Schnitzler F, Fidder $H$, Ferrante $M$, et al. Long-term outcome of treatment with infliximab in 614 patients with Crohn's disease: results from a singlecentre cohort. Gut 2009; 58:492-500.

27. Munkholm P, Langholz E, Davidsen M, Binder V. Frequency of glucocorticoid resistance and dependency in Crohn's disease. Gut 1994; 35:360-2.

28. Faubion WA Jr, Loftus EV Jr, Harmsen WS, Zinsmeister AR, Sandborn WJ. The natural history of corticosteroid therapy for inflammatory bowel disease: a population-based study. Gastroenterology 2001; 121:255-60.

29. D'Haens G, Geboes K, Rutgeerts P. Endoscopic and histologic healing of Crohn's (ileo-) colitis with azathioprine. Gastrointest Endosc 1999; 50:667-71.

30. Murakami Y, Matsui T, Hirai F, et al. Efficacy of azathioprine in mild or moderate relapse in Crohn's disease: clinical and endoscopic evaluation. Dig Endosc 2010; 22:25-32.

31. Kozarek RA, Patterson DJ, Gelfand MD, et al. Methotrexate induces clinical and histologic remission in patients with refractory inflammatory bowel disease. Ann Intern Med 1989; 110:353-6

32. Laharie D, Reffet A, Belleannée G, et al. Mucosal healing with methotrexate in Crohn's disease: a prospective comparative study with azathioprine and infliximab. Aliment Pharmacol Ther 2011; 33:714-21.

33. Rutgeerts $P$, Feagan BG, Lichtenstein GR, et al. Comparison of scheduled and episodic treatment strategies of infliximab in Crohn's disease. Gastroenterology 2004; 126:402-13.

34. Hanauer SB, Feagan BG, Lichtenstein GR, et al. Maintenance infliximab for Crohn's disease: the ACCENT I randomised trial. Lancet 2002; 359:1541-9.

35. Colombel JF, Sandborn WJ, Reinisch W, et al. Infliximab, azathioprine, or combination therapy for Crohn's disease. N Engl J Med 2010; 362:1383-95.

36. Rutgeerts P, Van Assche G, Sandborn WJ, et al. Adalimumab induces and maintains mucosal healing in patients with Crohn's disease: data from the EXTEND trial. Gastroenterology 2012; 142:1102-11.e2.

37. Hébuterne $X$, Lémann $M$, Bouhnik $Y$, et al. Endoscopic improvement of mucosal lesions in patients with moderate to severe ileocolonic Crohn's disease following treatment with certolizumab pegol. Gut 2013; 62:201-8.

38. Sipponen T, Björkesten CG, Färkkilä M, et al. Faecal calprotectin and lactoferrin are reliable surrogate markers of endoscopic response during Crohn's disease treatment. Scand J Gastroenterol 2010; 45:325-31.

39. Schoepfer AM, Beglinger C, Straumann A, et al. Fecal calprotectin correlates more closely with the Simple Endoscopic Score for Crohn's disease (SESCD) than CRP, blood leukocytes, and the CDAI. Am J Gastroenterol 2010; 105:162-9.

40. Sipponen T, Savilahti E, Karkkainen P, et al. Fecal calprotectin, lactoferrin, and endoscopic disease activity in monitoring anti-TNF-alpha therapy for Crohn's disease. Inflamm Bowel Dis 2008;14:1392-8.

41. Kolho KL, Raivio T, Lindahl H, Savilahti E. Fecal calprotectin remains high during glucocorticoid therapy in children with inflammatory bowel disease. Scand J Gastroenterol 2006; 41:720-5.

42. Louis E, Mary JY, Vernier-Massouille G, et al. Maintenance of remission among patients with Crohn's disease on antimetabolite therapy after infliximab therapy is stopped. Gastroenterology 2012; 142:63-70.e5.

43. Orlando A, Modesto I, Castiglione F, et al. The role of calprotectin in predicting endoscopic post-surgical recurrence in asymptomatic Crohn's disease: a comparison with ultrasound. Eur Rev Med Pharmacol Sci 2006; 10:17-22.

44. Panés J, Bouzas R, Chaparro M, et al. Systematic review: the use of ultrasonography, computed tomography and magnetic resonance imaging for the diagnosis, assessment of activity and abdominal complications of Crohn's disease. Aliment Pharmacol Ther 2011; 34:125-45.

45. Rimola J, Ordás I, Rodriguez S, et al. Magnetic resonance imaging for evaluation of Crohn's disease: validation of parameters of severity and quantitative index of activity. Inflamm Bowel Dis 2011; 17:1759-68.

46. Rimola J, Ordás I, Rodríguez S, Panés J. Colonic Crohn's disease: value of magnetic resonance colonography for detection and quantification of disease activity. Abdom Imaging 2010; 35:422-7.

47. Rimola J, Rodriguez S, García-Bosch O, et al. Magnetic resonance for assessment of disease activity and severity in ileocolonic Crohn's disease. Gut 2009; 58:1113-20.

48. Koilakou S, Sailer J, Peloschek P, et al. Endoscopy and MR enteroclysis: equivalent tools in predicting clinical recurrence in patients with Crohn's disease after ileocolic resection. Inflamm Bowel Dis 2010; 16:198-203. 